\title{
Primary Pulmonary Mucosa-Associated Lymphoid Tissue Lymphoma with a Nodular Opacity: Report of a Case
}

\author{
Naoyuki Yoshino ${ }^{1}$, Tomomi Hirata ${ }^{1}$, Chie Takeuchi ${ }^{1}$, \\ Jitsuo Usuda ${ }^{2}$ and Masaru Hosone ${ }^{3}$ \\ ${ }^{1}$ Department of Thoracic Surgery, Nippon Medical School Tama Nagayama Hospital, Tokyo, Japan \\ ${ }^{2}$ Department of Thoracic Surgery, Nippon Medical School, Tokyo, Japan \\ ${ }^{3}$ Department of Pathology, Nippon Medical School Tama Nagayama Hospital, Tokyo, Japan
}

\begin{abstract}
Herein, we describe our experience in treating a case of primary pulmonary mucosa-associated lymphoid tissue lymphoma detected as a nodular opacity. A 79-year-old man was referred to our hospital. Computed tomography showed a nodular opacity measuring $20 \mathrm{~mm}$ in diameter with regular margins in segment 5 of the right middle lobe of the lung. Although the bronchoscopic brush cytology result was class III, the patient was tentatively diagnosed with suspected mucosa-associated lymphoid tissue lymphoma. A thoracoscopic right middle lobectomy was performed. The pathological findings showed nodular proliferation of small to medium-sized, mature-appearing atypical lymphoid cells, lymphoepithelial lesions, and vague follicles suggesting follicular colonization in some areas. The patient was diagnosed with low-grade small B-cell lymphoma and mucosa-associated lymphoid tissue lymphoma. He has remained well to date, 23 months after surgery, without evidence of recurrence.
\end{abstract}

(J Nippon Med Sch 2017; 84: 87-89)

Key words: primary pulmonary lymphoma (PPL), mucosa-associated lymphoid tissue lymphoma (MALT), nodular opacity

\section{Introduction}

Extranodal lymphomas are most frequently seen in the gastrointestinal tract, but rarely in the lung. On roentgenographic images, primary pulmonary lymphomas (PPLs) usually appear as an alveolar mass or an infiltrate with ill-defined margins and air bronchograms ${ }^{1}$. These malignancies are relatively less likely to appear as nodular opacities. A bronchoscopy was formerly performed in most PPL patients but had a low diagnostic yield due to the infrequency of endoluminal lesions. ${ }^{1}$. We report our experience with a case of primary pulmonary mucosaassociated lymphoid tissue (MALT) lymphoma detected as a nodular opacity that with a diagnosis by bronchoscopy.

\section{Case Report}

Chest computed tomography (CT) detected a nodule in the right lung of a 79-year-old man before surgery of cataract at our institution. Because lung cancer was suspected, he was referred to the Department of Respiratory Medicine. The CT scan showed a nodule measuring 20 $\mathrm{mm}$ in diameter with relatively regular margins in segment 5 of the right middle lobe of the lung (Fig. 1). Although the bronchoscopic brush cytology result was class III, the patient was tentatively diagnosed with MALT lymphoma. A ${ }^{18} \mathrm{~F}$-fluorodeoxyglucose (FDG) positron emission tomography (PET) reveald a high FDG uptake (maximum standardized uptake value, 4.73-5.87 in the late phase). A thoracoscopic right middle lobectomy was performed. The pathological findings denoted nodular proliferation of medium-sized, mature-appearing atypical lymphoid cells, lymphoepithelial lesions, and vague follicles suggesting follicular colonization in some areas (Fig. 2). As determined via immunohistochemistry, the tumor cells expressed CD20, CD79a, PAX5, and BCL2 but did not express CD3, CD10, CD21, CD23, multiple myeloma

Correspondence to Naoyuki Yoshino, Department of Thoracic Surgery, Nippon Medical School Tama Nagayama Hospital, 1-7-1

Nagayama, Tama, Tokyo 206-8512, Japan

E-mail: yoshinoy@nms.ac.jp

Journal Website (http://www2.nms.ac.jp/jnms/) 

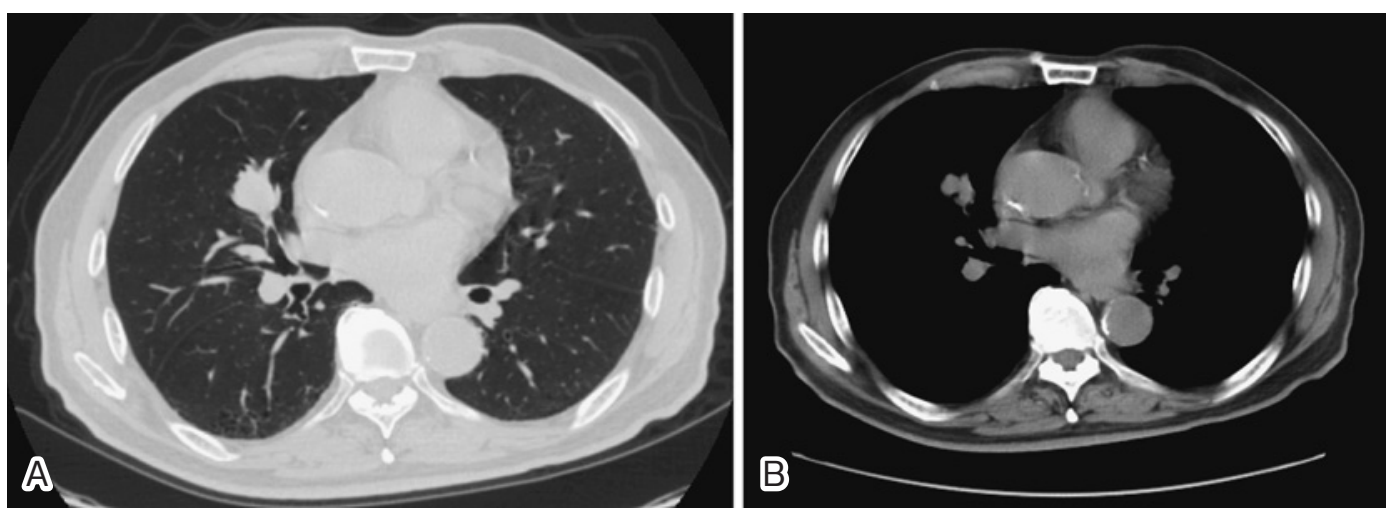

Fig. 1 Chest CT shows a nodule measuring $18 \times 18 \mathrm{~mm}$, with regular margins near the center of Segment 5 of the right middle lobe of the lung. (A: Lung field window B: Mediastinal window).

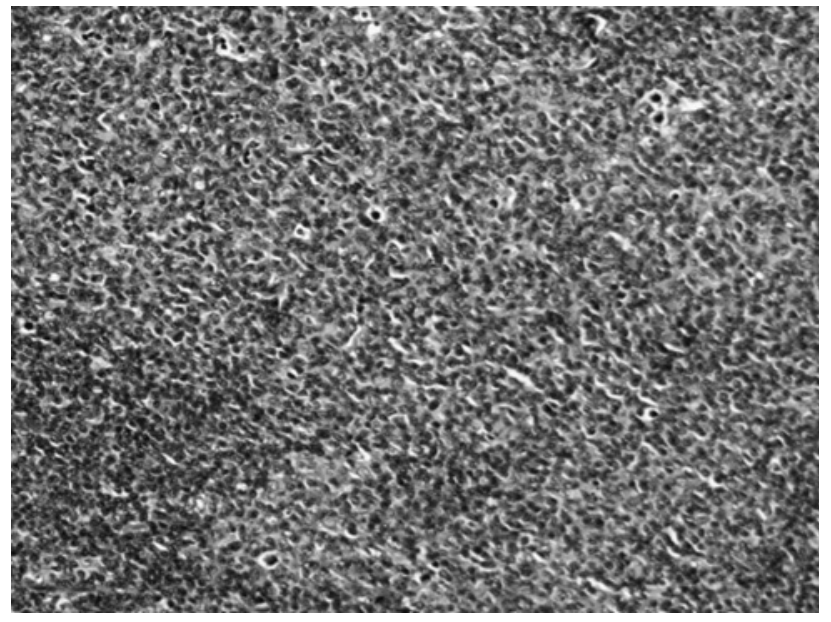

Fig. 2 Nodular proliferation of small or medium, matureappearing atypical lymphoid cells is noted. In some areas, lymphoepithelial lesions as well as vague follicles suggesting follicular colonization are observed $(\mathrm{HE} \times 100)$.

oncogene 1, cyclin D1, or BCL6. The proliferative index determined using the MIB-1 antibody was approximately $10 \%$. The patient was diagnosed with small B-cell lymphoma and MALT lymphoma. Preoperative FDG-PET showed no FDG uptake in any organs other than the lung. Five months postoperatively, gallium scintigraphy showed no abnormal gallium accumulation. The patient has remained well to date, 23 months after surgery, without evidence of recurrence. Thus, our case was diagnosed a primary pulmonary MALT lymphoma.

\section{Discussion}

Most PPLs arise from MALT in the bronchus². MALT lymphoma is an extranodal non-Hodgkin B-cell lymphoma generated from MALT in various organs. It is now categorized as an extranodal, marginal zone, B-cell lymphoma according to the revised European-American classification of lymphoid neoplasms ${ }^{2}$. PPLs represent less than $1 \%$ of lung cancers and malignant lymphomas, and only $3.6 \%$ of extranodal lymphomas ${ }^{3}$.

The definition of the most-used PPL proposed by L'Hoste et al. is a lymphoma that involves the lung lobar, or primary bronchus, with or without mediastinal involvement. In addition, lymphoma outside the chest is not proved for 3 months after diagnosis ${ }^{4}$.

On radiographic images, PPL appears as a nodule or mass, an ill-defined infiltrate or amalgam without any dominant feature ${ }^{5}$. Roentgenographic scans typically show a pulmonary infiltrate with diffuse margins and air bronchograms $(69 \%)$ or an alveolar mass $(60 \%)^{1}$. Bronchioloalveolar carcinoma, cryptogenic organizing pneumonia, metastases and lymphocytic interstitial pneumonia, will be cited as a radiological differential diagnoses of PPL ${ }^{6}$. These findings suggest that any radiological abnormality of the lung parenchyma represents a possible lymphoma ${ }^{5}$. Peribronchial disease, proximal bronchiectasis, and a positive CT-angiogram sign are ancillary features of PPLs, and magnetic resonance imaging may be useful for evaluation of pleural or chest wall involvement $^{6}$. According to Kocatürk et al., PPL shows minimal uptake of FDG on PET. Hence, the benefit of FDG-PET in the clinical assessment of PPL is controversial ${ }^{7}$. However, in our case, FDG accumulated in the nodule, and FDGPET was useful for diagnosing PPL.

Radiologic images are not specific, and the diagnostic capability of the bronchoscopy are also limited, diagnosis is often made by surgical resection ${ }^{8}$. Ferraro et al. reported that bronchoscopy was performed in 39 patients with PPLs but confirmed the diagnosis in only seven pa- 
tients $(18 \%)^{1}$. Molecular biological techniques such as flow cytometry using bronchoalveolar lavage fluid may be helpful in diagnosing $\mathrm{PPL}^{5}$. In our case, cytological specimens from transbronchial-brushing contributed to the definitive diagnosis. Bronchoscopy may be useful when malignant PPL is suspected on the basis of imaging findings, and flow cytometry analysis of bronchoalveolar lavage fluid should be performed.

Treatments for pulmonary MALT lymphoma include surgery, chemotherapy, and radiotherapy; however, no therapeutic methods have yet been established. Excising the lesion, by diagnostic lung resection employing wedge resection or lobectomy, is often the only treatment. When a patient is diagnosed as having pathologically lowgrade MALT lymphoma but has no other lesions, complete excision of the lesion is recommended, and no further treatment should be necessary. When there are residual lesions, chemotherapy or radiotherapy (in cases with localized lesions) should be considered. In our case, the MALT lymphoma was considered to have been completely removed, and the clinical course was observed without postoperative adjuvant chemotherapy.

The prognosis of pulmonary MALT lymphoma is reportedly favorable. Ferraro et al. reported that the survival rate of patients with this disease is $91 \%$ at one year, $68 \%$ at five years, and $53 \%$ at 10 years $^{1}$, while Kurtin et al. obtained corresponding rates of $95 \%, 84.5 \%$, and $71.1 \%$. However, Kurtin et al. stated that the survival curve for MALT lymphoma never reaches a plateau, and that the risks of recurrence or death from the primary pulmonary MALT lymphoma persist indefinitely'.

The prognostic factors that affect survival of MALT lymphoma has not yet been defined. One of the probable factors affecting survival is histopathological diagnosis.

According to Kocatürk et al., some studies have demonstrated poorer prognosis of non-MALT lymphomas, but the difference in survival rate of MALT lymphomas and non-MALT lymphomas was not observed ${ }^{7}$.

In our case, a nodular opacity with regular margins was detected by radiography. This was recognized as an atypical imaging finding. We believe PPL should be con- sidered if images similar to our case. Diagnosis of PPL via bronchoscopy is not easily accomplished. In our case, the results of a preoperative bronchoscopy suggested lymphoma, and the presence of this disease type was thereby inferred. When radiological abnormalities of the lung parenchyma are detected, it might be advisable to perform bronchoscopy even if lymphoma is suspected.

Conflict of Interest: No conflict of interest is declared.

\section{References}

1. Ferraro P, Trastek VF, Adlakha H, Deschamps C, Allen MS, Pairolero PC: Primary non-Hodgkin's lymphoma of the lung. Ann Thorac Surg 2000; 69: 993-997.

2. Kawashima T, Nishimura H, Akiyama H, Hirai K, Yamagishi S, Okada D, Kinoshita H, Enomoto Y, Okamoto J, Nakajima Y, Takeuchi S, Iijima Y, Furuhata K, Nakayama K, Izumo T, Koizumi K, Shimizu K: Primary pulmonary mucosa-associated lymphoid tissue lymphoma combined with idiopathic thrombocytopenic purpura and amyloidoma in the lung. J Nippon Med Sch 2005; 72: 370-374.

3. Graham BB, Mathisen DJ, Mark EJ, Takvorian RW: Primary pulmonary lymphoma. Ann Thorac Surg 2005; 80: 1248-1253.

4. L'Hoste RJ Jr, Filippa DA, Lieberman PH, Bretsky S: Primary pulmonary lymphomas. A clinicopathologic analysis of 36 cases. Cancer 1984; 54: 1397-1406.

5. Kim JH, Lee SH, Park J, Kim HY, Lee SI, Park JO, Kim K, Kim WS, Jung CW, Park YS, Im YH, Kang WK, Lee MH, Park K, Han JH, Ko YH: Primary pulmonary nonHodgkin's lymphoma. Jpn J Clin Oncol 2004; 34: 510-514.

6. Ooi GC, Chim CS, Lie AK, Tsang KW: Computed tomography features of primary pulmonary non-Hodgkin's lymphoma. Clin Radiol 1999; 54: 438-443.

7. Kocatürk Cİ, Seyhan EC, Günlüoğlu MZ, Urer N, Kaynak $\mathrm{K}$, Dinçer Sİ, Bedirhan MA: Primary pulmonary nonHodgkin's lymphoma: ten cases with a review of the literature. Tuberk Toraks 2012; 60: 246-253.

8. Vanden Eynden F, Fadel E, de Perrot M, de Montpreville $\mathrm{V}$, Mussot S, Dartevelle P: Role of surgery in the treatment of primary pulmonary B-cell lymphoma. Ann Thorac Surg 2007; 83: 236-240.

9. Kurtin PJ, Myers JL, Adlakha H, Strickler JG, Lohse C, Pankratz VS, Inwards DJ: Pathologic and clinical features of primary pulmonary extranodal marginal zone B-cell lymphoma of MALT type. Am J Surg Pathol 2001; 25: 997-1008.

(Received, January 26, 2016)

(Accepted, February 12, 2016) 